\title{
Use of Trichloroacetic Acid in Treating Facial Hyperpigmentation
}

\author{
RADU CRISTIAN JECAN ${ }^{1,2,3}$, ALEXANDRU NICOLAU4*, IOAN PETRE FLORESCU1,3,5, VALERIU ARDELEANU1,4, SORIN BERBECE ${ }^{4}$ \\ ${ }^{1}$ Arestetic Clinic Galati, 78 Brailei Str., 800098, Galati, Romasnia \\ ${ }^{2}$ Agripa Ionescu Emergency Clinical Hospital Bucharest, 7 Ion MIncu Str., 011356, Bucharest, Romania \\ ${ }^{3}$ Carol Davila University of Medicine and Pharmacy, 8 Eroii Sanitari Str., 050474, Bucharest, Romania \\ ${ }^{4}$ Dunarea de J os University of Galati, Faculty of medicine and Pharmacy, 47 Domneasca Str., 800008, Galati, Romania \\ ${ }^{5}$ Bagdasar Arseni Clinical Emergency Hospital, 12 Berceni Road, 041915, Bucharest, Romania
}

\begin{abstract}
This paper aims to present the benefits and results of using TCA of high concentration of $50 \%$, in conjunction with the presentation of adverse reactions and complications. The study was conducted in Arestetic Clinic Galai during the period 10 Dec. 2015-1 Nov. 2016, on a group of 12 patients, of which 10 women and 2 men. The product used was Easy TCA 50\% manufactured by SkinTech laboratories. Three coats of TCA were applied at intervals of 4 minutes each. The end result was assessed at 21 days, when the entire surface of the skin was peeled and re-epithelized. Patients were asked to assess the degree of satisfaction on a scale of 1 to 10, with 1 being the least satisfactory and 10 being the most satisfactory. As a result of this assessment, 10 patients rated the degree of satisfaction with $10(83 \%)$ and 2 patients rated the degree of satisfaction with 9(17\%), with an average degree of satisfaction of 9.83 . At 21 days, dyschromias and hyperpigmentations completely disappeared in 9 patients, while a female patient and 2 male patients still presented with barely noticeable spots. These three persons were subjected to the second peeling after 2 months, this time in two layers, and these hyperpigmentations disappeared at the end.
\end{abstract}

Keywords: peeling, hyperpigmentation, trichloroacetic acid

The chemical peeling represents an aesthetic process which consists of applying acid solutions of different concentrations for superficial skin layer removal and skin rejuvenation. The main uses are represented by skin hyperpigmentations and fine wrinkles, butitcan be applied to acne and post-acne scars or dilated pores. Chemical peels can be performed with alpha hydroxy acids or beta hydroxy acids and, according to the desired peeling depth, these can be superficial, medium or deep. Trichloroacetic acid (TCA) can be used both for superficial chemical peels in concentrations of $15 \%$, as well as for medium chemical peels in concentrations of 35\% and $50 \%$. The mechanism of action on the skin is that of protein coagulation or keratocoagulation at the level of the epidermis and the dermis. Due to the superficial skin layers destructuring and the stratum corneum removal, the epidermis, the collagen, the elastin, and the glycozaminoglycans growth will be stimulated. As a consequence, it will result in the recovery of the superficial layers, resulting in a global skin rejuvenation. This paper aims to present the benefits and results of using TCA in a high concentration of $50 \%$, in conjunction with the presentation of adverse reactions and complications.

\section{Experimental part}

Material and method

The study was conducted in Arestetic Clinic Galati during the period 10 Dec. 2015-1 Nov. 2016, on a group of 12 patients, of which 10 women and 2 men. The age ranged between 32 and 57 years old, with an average age of 46 years.

The product used was Easy TCA 50\% from SkinTech laboratories.

The work method was the following: $0.7 \mathrm{~mL}$ TCA $50 \%$ diluted in $2 \mathrm{~mL}$ of base solution was used for each patient. 3 layers were applied at intervals of 4 minutes each, as follows:
- the first layer: the face was divided into 7 areas. For each area, the cotton bud was soaked in the peeling solution (Photo 1).

- the second layer: the face was divided into three areas, the cotton buds being soaked in the TCA solution (Photo 2) for each area.

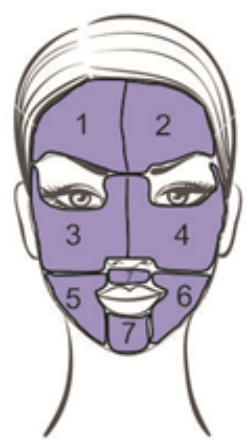

Photo 1

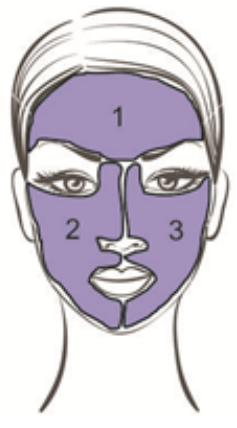

Photo 2
- the third layer: the pattern for the second layer was used, but, at the same time, the aim was to obtain an even frost to prevent subsequent facial dyschromias.

The aim for the first layer was the appearance of an erythema or points of frost. The aim for the second layer was to obtain the frost clouds. The aim for the third layer was to obtain a full quasi-uniform ivory-white frost.

After 4 minutes from the third layer, the neutralization mask was applied for $45 \mathrm{~min}$. The reassessment of the patient was performed at 3,7 and 14 days. The final result was determined after a period of 14 days. During this period, the patients received an SPF 50 cream and the recommendation to avoid the exposure to the sun, the snow or the sun room. The adjuvant was used in days 3-7, as well as moisturizing creams. 


\section{Results and discussions}

After applying the first layer of TCA, we obtained an intense erythema and points of frost, associated with a slight burning sensation, in all the patients. After applying the second layer of TCA, we obtained the frost clouds amid the intensification of the burning sensation. With the third layer, we wanted to even the frost in order not to create facial dyschromia after the peeling. In assessing the discomfort state, the patients were asked to assess the discomfort state on a scale of 1 to 10 , with 1 being the least disturbing and 10 being the most disturbing. Following this assessment, the average degree of discomfort was 7.91.

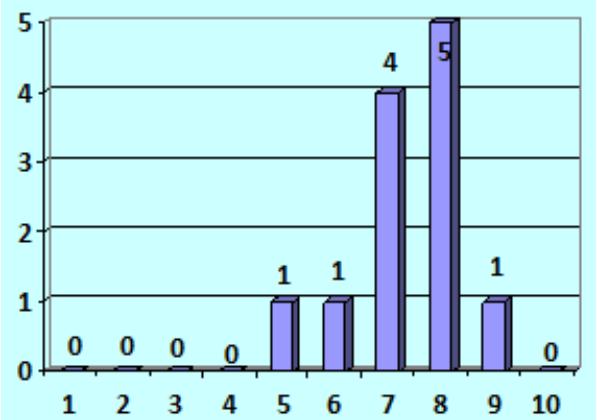

After applying the neutralization mask, the face became slightly red after 1 hour, without any feeling of discomfort. In the first 3-4 days, the skin spots intensified in colour, becoming dark brown. Starting with day 4-5, the skin began to peel, lasting up to 10-12 days. The end result was assessed after 21 days, when the entire surface of the skin was peeled and re-epithelized. The patients were asked again to assess the degree of satisfaction on a scale of 1 to 10 , with 1 being the least satisfactory and 10 being the most satisfactory. Following this assessment, 10 patients rated the degree of satisfaction with $10(83 \%)$ and 2 patients rated the degree of satisfaction with $9(17 \%)$, with an average degree of satisfaction of 9.83 . We mention that both assessments of 9 were given by the male persons.

As an aim after 21 days, the dyschromias and hyperpigmentations completely disappeared in 9 patients, while a female patient and 2 male patients still presented with barely noticeable spots. These three persons were subjected to the second peeling after 2 months, this time in two layers, and these hyperpigmentations disappeared at the end.

The post-procedural results within the range of chemical peels vary in direct proportion to the level of the depth at which they are carried out and the degree of experience of the medical procedure provider $[2,6]$.

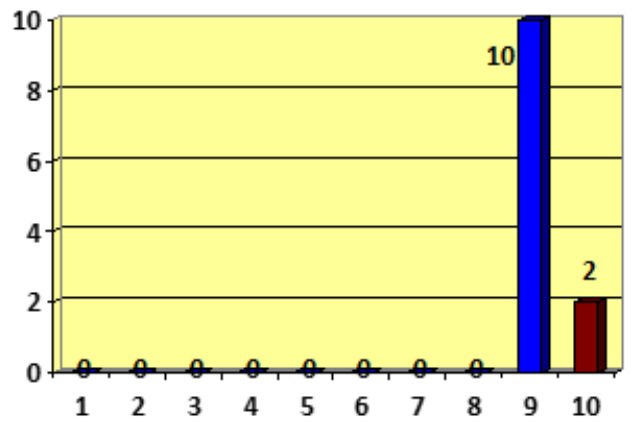

The principle of chemical skin peel consists of the skin regeneration ability to prevent the controlled iatrogenic injuries, so that the healing potential should be maximized at the expense of scar damage potential $[2,4]$.

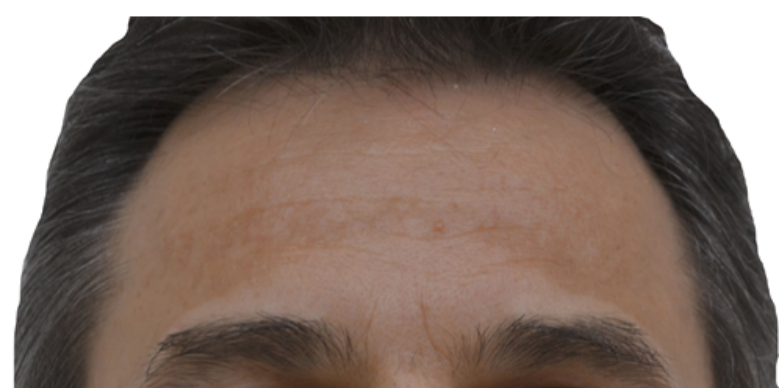

Photo 3. Patient 1: before TCA peeling

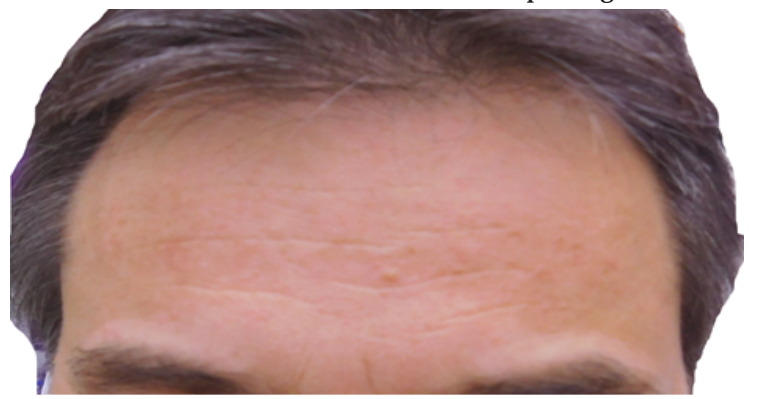

Photo 4. Patient 1: at 3 weeks after 2 TCA peeling

In this context, the use of high concentrations of up to $50 \%$ of trichloroacetic acid solution facilitates the access to the remedy of superficial cutaneous structures, even deep environments having as starting point the reticular dermis [7].Thus, through the keratocoagulating property, it induces necrosis and exfoliation of keratinocytes found in a slow, normal or aberrant evolutionary cycle, becoming extremely useful in treating the epidermal disorders, such as the seborrheic keratosis and the acne keratosis, as the common warts or milia. By interfering with the basal epidermal membrane structures and with adjacent melanin deposits, it allows the removal of the skin dyschromia, such as melasma [3], ephelides, lentigos or post-inflammatory hyperpigmentations [1, 2].

The restoration of the basal skin membrane, along with reaching the reticular dermis, means the reshaping of collagen and the scar contracture phenomenon, which emphasizes the applicability of this procedure in the treatment of superficial wrinkles and aging process related environments [2, 8].

Although the best results are obtained after a deep chemical peel, this procedure can generate powerful consequences while performed by unexperienced providers. The inappropriate choice of trichloroacetic acid concentration or its action time, relative to the area treated, can lead to significant side effects [5]. The complications incidence is directly proportional to the depth at which the procedure is performed, which in turn, is directlyinfluenced by the concentration of the product used [4,6]. Identifying ideal candidates for this type of procedure plays a defining role in obtaining optimum results [7].

Thus for patients with closed phototypes, Fitzpatrik III$\mathrm{VI}$, who present an additional risk of pathological scarring or developing dyschromic phenomena, low concentrations (10-25\%) shall always be used $[2,4,6]$.

\section{Conclusions}

TCA 50\% chemical peel is a safe peeling, highly effective discoloration, and correctly applied not give complications.

\section{References}

1.KUMARI R, THAPPA DM. - Comparative study of trichloroacetic acid versus glycolic acid chemical peels in the treatment of melasma. Indian J Dermatol Venereol Leprol. 2010 J ul-Aug; 76(4):447. doi: 10.4103/ 0378-6323.66602 
2.KHUNGER N; IADVL Task Force - Indian J Dermatol Venereol Leprol. 2008 Jan;74 Suppl:S5-12.Standard guidelines of care for chemical peels

3.*** Chemical peeling-glycolic acid versus trichloroacetic acid in melisma - Kalla G, Garg A, Kachhawa D.

4.CAMACHO. Medium-depth and deep chemical peels.. FM Cosmet Dermatol. 2005 Jun;4(2):117-28.

5.FUNG JF, SENGELMANN RD, KENNEALLY CZ.Chemical injury to the eye from trichloroacetic Acid. Dermatol Surg. 2002 Jul;28(7):609-10; discussion 610.
6.KIMURA A, KANAZAWA N, LI HJ , YONEI N, YAMAMOTO Y, FURUKAWA

F.Influence of chemical peeling on the skin stress response system. Exp Dermatol. 2012 Jul;21 Suppl 1:8-10. doi: 10.1111/j.16000625.2012.01495.x

7. DOGRA A, GUPTA S, GUPTA S. Comparative efficacy of $20 \%$ trichloroacetic acid and $50 \%$ glycolic acid peels in treatment of recalcitrant melisma. J Pak Assoc Derma 2006; 15:79-85

8. Monheit Go. Chemexfoliation: A review. Cosmetic Dermatology 1988;1:16-19.

Manuscript received: 15.12 .2016 